\title{
Neuroinflammatory astrocytes generated from cord blood-derived human induced pluripotent stem cells
}

\author{
Qiong Zhou', Coralie Viollet' ${ }^{2}$, Anastasia Efthymiou ${ }^{3}$, Guzal Khayrullina ${ }^{4}$, Kasey E. Moritz ${ }^{5}$, Matthew D. Wilkerson ${ }^{6}$, \\ Gauthaman Sukumar ${ }^{6}$, Clifton L. Dalgard ${ }^{1,4,6}$ and Martin L. Doughty ${ }^{1,4^{*}}$ (D)
}

\begin{abstract}
Background: Astrocytes respond to central nervous system (CNS) injury and disease by transforming to a reactive astrogliosis cell state that can contribute to either CNS dysfunction or repair. Neuroinflammation is a powerful driver of a harmful A1 astrogliosis phenotype associated with in vitro neurotoxicity and histopathology in human neurodegenerative diseases. Here we report a protocol for the rapid development of a human cell culture model of neuroinflammatory astrogliosis using induced pluripotent stem cells (iPSCs).

Methods: Using RNA sequencing and in vitro cell assays, we measured transcriptional and cellular effects of chronic exposure of human iPSC-derived astrocytes to the cytokines TNFa (tumor necrosis factor alpha) or IL-1 $\beta$ (interleukin-1 beta).

Results: We show TNFa and IL-1 $\beta$ induce pro-inflammatory gene signatures but by widely different magnitudes. TNFa treatment results in 606 differential expressed genes, the suppression of glutamate-uptake, and increased phagocytic activity in astrocyte cultures. In contrast, IL-1 $\beta$ effects are attenuated to 33 differential expressed genes and no significant effects on glutamate-uptake or increased phagocytic activity.

Conclusion: Our approach demonstrates a rapid tool for modeling neuroinflammatory human astrocytic responses in nervous system trauma and disease. In particular, we reveal a model for robust TNFa-induced human astrogliosis suitable for the study of neurotoxic A1 astrocytes.
\end{abstract}

Keywords: Human induced pluripotent stem cell, Astrocyte, RNA sequencing, Glutamate uptake, Phagocytosis

\section{Highlights}

Astrocytes rapidly differentiated from neural stem progenitor cells (NSPCs) of human cord blood-derived iPSC origin are differentially sensitive to neuroinflammatory cytokines. Chronic TNF $\alpha$ treatment results in widespread differential gene expression, suppressed glutamate uptake, and increased phagocytic activity in cultured iPSC-derived astrocytes. In contrast, IL- $1 \beta$ effects are attenuated with few differentially expressed genes and no effect on glutamate uptake or phagocytic activity.

\footnotetext{
* Correspondence: martin.doughty@usuhs.edu

${ }^{1}$ Center for Neuroscience and Regenerative Medicine, Uniformed Services University of the Health Sciences, Bethesda, MD 20814, USA

${ }^{4}$ Department of Anatomy, Physiology and Genetics, Center for Neuroscience and Regenerative Medicine, Uniformed Services University of the Health Sciences, Bethesda, MD 20814, USA

Full list of author information is available at the end of the article
}

\section{Background}

Astrocytes are abundant cells in the CNS contributing $\sim 40 \%$ of all cells in the human brain [1]. Astrocytes function in a range of neurodevelopmental and homeostatic processes in the brain that include the establishment and maintenance of synapses [2]; the regulation of energy supply [3], of extracellular neurotransmitter levels [4], and of brain blood flow [5]; and the maintenance of the blood-brain barrier [6].

Consistent with their wide-ranging homeostatic role, astrocyte dysfunction is linked to a number of neurodevelopmental disorders such as autism spectrum disorder [7] and Down's syndrome [8] and neurodegenerative diseases such as Alzheimer's [9] and Parkinson's disease [10]. Astrocytes are acutely sensitive to changes in their cellular environment and react to CNS damage and

(c) The Author(s). 2019 Open Access This article is distributed under the terms of the Creative Commons Attribution 4.0 International License (http://creativecommons.org/licenses/by/4.0/), which permits unrestricted use, distribution, and 
disease with changes in gene expression, morphology, and function. This reactive astrocyte state is referred to as astrogliosis [11].

Astrogliosis is a heterogeneous, stimulus-dependent cell state. Middle cerebral artery (MCA) occlusion or lipopolysaccharide (LPS) induced neuroinflammation drive transcriptionally distinct astroglia [12] characterized by divergent neuroprotective or neurotoxic phenotypes respectively [13]. Neurotoxic astroglia, also referred to as A1 astrocytes, are induced by LPS-activated microglia through microglial secretion of the neuroinflammatory cytokines TNF $\alpha, \mathrm{IL}-1 \alpha$, and the complement component C1q [13]. LPS also induces increased IL-1 $\beta$ secretion by neuroinflammatory microglia, but unlike TNF $\alpha$, IL-1 $\alpha$, or C1q, IL-1 $\beta$ treatment alone does not induce astrocyte expression of A1 associated transcripts. These data reveal differential sensitivity to the classical neuroinflammatory cytokines TNF $\alpha$ and IL-1 $\beta$ in astrocytes.

Although rodent animal and cell culture models have provided important data on astrocyte neuroinflammatory signaling, increasing evidence suggests human astrocytes are morphologically, transcriptionally, and functionally distinct from those of rodents. Human astrocytes are morphologically larger and more complex than their rodent counterparts [14], and RNA sequencing of acutely isolated astrocytes reveals a gene expression signature unique to human astrocytes [1]. Xenotransplantation of human astrocytes into the brains of developing mice enhances behavioral learning and long-term potentiation (LTP) in the hippocampus with engrafted human astrocytes exhibiting accelerated $\mathrm{Ca}^{2+}$ wave propagation compared to host astrocytes [15]. Furthermore, human astrocytes retain their complex morphology and distinct $\mathrm{Ca}^{2+}$ responses when stimulated in vitro [1]. Combined, these data highlight the importance and feasibility of adopting human astrocyte model culture systems to investigate astrocyte pathology in brain trauma and disease.

Induced pluripotent stem cell (iPSC)-derived astrocytes offer an increasingly popular method for modeling physiological and pathological human astrocyte functions in culture $[8,16-21]$. Although this approach remains hampered by gaps in our understanding of the signaling events that drive topographical and functional subtype specificity in astrocytes, human iPSC-derived astrocytes have revealed novel pathological features in neurodevelopmental disorders [8] and neurodegenerative disease [21]. In this study, we characterize the neuroinflammatory responses of astrocytes rapidly derived from neural stem progenitor cells (NSPCs) from the human iPSC line NCRM-1. NCRM-1 iPSCs were generated from CD34+ human cord blood cells by episomal plasmid reprogramming [22] and differentiated to positional naïve NSPCs through an embryoid body (EB) method
[23]. NSPCs were differentiated to astrocytes as previously described $[16,17]$ and treated with pro-inflammatory cytokines TNF $\alpha$ or IL-1 $\beta$. Expression profiling and in vitro glutamate uptake and phagocytosis assays reveal a robust astrocyte inflammatory response to TNF $\alpha$. In contrast, IL-1 $\beta$ effects are attenuated. We conclude our approach reveals a rapid and efficient protocol for modeling A1-like TNF $\alpha$ inflammatory responses in human astrocytes in CNS disease or trauma.

\section{Methods \\ Human iPSC-derived NSPC cell culture and astrocyte differentiation}

In this study, we use neural stem progenitor cells (NSPCs) derived from the human induced pluripotent stem cell (iPSC) line NCRM-1 [24, 25]. NCRM-1 iPSCs were generated in collaboration with the NIH, Sigma-Aldrich (now MilliporeSigma), and XCell Sciences from CD 34+ human cord blood cells by episomal plasmid reprogramming https://www.nimhgenetics.org/stem_cells/ crm_lines.php. NCRM-1 iPSCs were differentiated to NSPCs through an embryoid body (EB) method by XCell Sciences. NSPCs were maintained as previously described [24] in Neural Stem Cell Medium (NSCM) on Geltrex ${ }^{\circ}$ (Life Technologies) coated plates. NSCM consisted of Neurobasal Medium, GlutaMAX ${ }^{\mathrm{in}}$, non-essential amino acids, B27 supplement (all from Life Technologies, Carlsbad, CA), and $10 \mathrm{ng} / \mathrm{mL}$ fibroblast growth factor-2 (FGF2) (R\&D Systems, Minneapolis, MN). Media was refreshed every other day, and cells were passaged at a 1:3 ratio once the cells reached $\sim 80 \%$ confluence.

NCRM-1 NSPCs were differentiated to astrocytes as previously described [16]. Briefly, NSPCs were passaged to $1 \times 10^{6}$ cells per well of a six-well plate. Media was changed to Astrocyte Differentiation Medium (ADM) after $24 \mathrm{~h}$. ADM contained DMEM/F12 supplemented with GlutaMAX ${ }^{\mathrm{x}}$, $1.8 \%$ BSA, 1x StemPro hESC Supplement (all from Life Technologies), $10 \mathrm{ng} / \mathrm{mL}$ ActivinA (R\&D Systems), $20 \mathrm{ng} / \mathrm{mL}$ Heregulin1b (R\&D Systems), 10 ng/mL IGF-1 (PeproTech, Rocky Hill, NJ), and 10 ng/mL FGF2 (R\&D) supplemented with $1 \%$ fetal bovine serum. Cells were passaged every 4 days at a 1:3 ratio. Cells used for imaging were passaged to Geltrex-coated coverslips 2 days before immunocytochemistry. Astrocyte fate was confirmed when $\approx 100 \%$ of the cells were CD $44+$ and $\approx 50 \%$ of the cells were GFAP+ (between 35 and 70 days in ADM).

\section{Cytokine treatment}

Drug treatment on NCRM-1 astrocytes was performed on cultures containing $\sim 100 \%$ CD $44+$ cells and $\geq 50 \%$ GFAP+ cells. Astrocytes were derived as described previously and plated onto Geltrex-coated 96-well plates after 35-50 days culture in astrocyte differentiation conditions. Astrocytes were treated with 10,30 , or $100 \mathrm{ng} / \mathrm{mL}$ of 
tumor necrosis factor alpha (TNF $\alpha$, PeproTech, Rocky Hill, NJ) or with 10,30 , or $100 \mu \mathrm{g} / \mathrm{mL}$ of interleukin-1 beta (IL-1 $\beta$, PeproTech) for 7 days. Controls were treated with PBS/BSA vehicle. The media was replaced with fresh media + cytokine/vehicle after 4 days of treatment.

\section{Western blot}

Whole-cell protein extracts were obtained from astrocyte cultures using NE-PER ${ }^{\circ}$ reagents (Thermo Scientific) according to the manufacturer's instructions. Protein fractions were stored at $-20^{\circ} \mathrm{C}$ in $1 \mathrm{x}$ Halt ${ }^{\mathrm{Tx}} / 1 \mathrm{x}$ Halt $^{\text {mi }}$ Phosphatase Inhibitor Cocktail (both Thermo Scientific) until use. Twenty micrograms of protein was loaded per lane and samples separated by SDS-PAGE and electro-transferred to PVDF membrane. For TNFR1 and IL-1R1 blots, human PBMC (gift of A. Snow) and human MOLT-4 (Abcam, Cambridge, MA) cell lysate samples were included as positive controls respectively. Washes and antisera incubations were performed in $5 \%$ skim milk or $5 \%$ BSA in TBS with $0.1 \%$ Tween-20. Blots were incubated with primary antisera overnight at $4{ }^{\circ} \mathrm{C}$ on a rocking platform. Primary antisera and dilutions used were as follows: 1:1000 rabbit polyclonal antiNF-к $\beta$ p65 (Cell Sig. Tech., Danvers, MA); 1:1000 rabbit phospho-anti-NF-к $\beta$ p65 (Ser536) (Cell Sig. Tech); 1: 1000 rabbit polyclonal anti-IL-1R1 (Abcam); 1:1000 rabbit polyclonal anti-TNF-R1 (Cell Sig. Tech); 1:1000 rabbit anti-Actb (Cell Sig. Tech); and 1:1000 mouse monoclonal anti-Gapdh (MilliporeSigma, St. Louis, $\mathrm{MO})$. The following day, blots were washed 3 times and incubated with a 1:5000 dilution of peroxidase-conjugated goat anti-mouse or anti-rabbit IgG secondary antisera (Cell Sig. Tech.) for $1 \mathrm{~h}$ at room temperature. Blots were developed in Immobilon chemiluminescent ECL substrate (Millipore) for $5 \mathrm{~min}$ at room temperature and the fluorescent signal captured using a BIO-RAD ChemiDoc Touch imaging system (BIO-RAD, Hercules, CA 94547). Western blot data was generated from three experimental replicates and immunosignal intensities (protein density) measured using BIO-RAD software. For each replicate, phospho-anti-NF- $\kappa \beta$ immunosignals were normalized to anti-NF- $\kappa \beta$ immunosignals and the densitometry data was presented as the normalized mean signal \pm SD.

\section{Transcriptome profiling by RNA sequencing}

Total RNA from harvested iPSC culture was quantified by fluorescence dye-based methodology (RiboGreen) on a Spectramax Gemini XPS plate reader (Molecular Devices, Mountain View, CA). RNA integrity was assessed using automated gel-based electrophoresis on an Experion automated electrophoresis system (Bio-Rad, Hercules, CA). Samples used as input for library preparation were RNA integrity number (RIN) value $>8$. Total
RNA input amount of $200 \mathrm{ng}$ was used for library preparation using the TruSeq Stranded mRNA Library Preparation Kit (Illumina, San Diego, CA). Sequencing library concentration was measured by quantitative PCR using KAPA Library Quantification Kit for NGS (Kapa, Wilmington, MA) on a CFX384 real-time PCR detection system (Bio-Rad, Hercules, CA) and assessed for size distribution on an Experion automated electrophoresis system (Bio-Rad). Sequencing libraries were pooled and sequenced on a NextSeq 500 desktop sequencer (Illumina) using a NextSeq 500 High Output v2 Kit with paired-end reads at $75 \mathrm{bp}$ length. Raw sequencing data was demultiplexed using bcl2fastq2 Conversion Software 2.17 before alignment using TopHat Alignment v1.0 and differential expression analysis using Cufflinks Assembly \& DE v1.1.0 on BaseSpace Onsite (Illumina) with the Illumina iGenomes UCSC hg19 reference and GTF transcript annotation files. Comparative analysis of transcript abundance values was performed using GenePattern 3.9 [26] with comparative marker selection features analyzed for gene ontology enrichment using PANTHER Classification System [27]. The Database for Annotation, Visualization and Integrated Discovery (DAVID) v6.8 was utilized for ontology and pathway enrichment analysis [28]. RNA sequencing was performed on a minimum three biological replicates for each treatment group.

\section{Real-time quantitative reverse transcription PCR (qRT- PCR)}

First-strand cDNA was synthesized from $1 \mu \mathrm{g}$ of total RNA using random primer/oligo ( $\mathrm{dT}$ ) primer according to the manufacturer's instructions (ABI, Carlsbad, CA). Synthesized cDNA was diluted to final concentration of $10 \mathrm{ng} / \mu \mathrm{l}$ for qPCR using SYBR Green Master Mix (ABI) on a Thermal cycler C1000 with CFX384 or CFX96 real-time system. Optimum primers were designed using NCBI's Primer BLAST www.ncbi.nlm.nih.gov/tools/primer-blast/. Primer sequence pairs used are detailed in Table 1 . Primer specificity was confirmed by verifying a single PCR product had been generated using UV gel electrophoresis, as well as by confirming the melting temperature of the product had a single value on dissociation plots. Relative expression levels were calculated using the Bio-Rad CFX Manager 3.1 software. Briefly, gene expression was normalized $(\Delta \mathrm{Cq})$ for each gene to the highest expression level by dividing the expression level of each sample by the highest level of expression in all the samples. The software sets the highest level of expression to a value of 1 and re-scales all the sample expression levels. The expression was then normalized $(\Delta \Delta \mathrm{Cq})$ to the 
Table 1 Primer sequences used for real-time quantitative reverse transcription PCR (qRT-PCR)

\begin{tabular}{lll}
\hline Gene & Forward primer & Reverse primer \\
\hline C3 & TAC AAC GTG GAG GCC ACA TC & ACG GGA GGC ACA AAG TCA AA \\
CCL2 & CAG CCA CCT TCA TTC CCC AA & GAC ACT TGC TGC TGG TGA TTC \\
CXCL10 & TGC ATC GAT TाT GCT CCC CT \\
ILCL11 1 1R1 & AAG ATC CAG AAT CGA AGG CCA TGA AAG TGG CAG AT & AAG CCT TGC TTG CTT CGA T \\
IL-8 & GGA GGA CTT GTG TGC CCT TA & CCA CAT TCA TCA CGA TGA GCC \\
MMP9 & AAG GTG CAG TTT TGC CAA GG & GTG TGG TCC ACT CTC AAT CAC T \\
TNFSR1 & GTA CTC GAC CTG TAC CAG CG AGCC ACT TCT TGT CG \\
\hline
\end{tabular}

quantities of reference house-keeping (HK) genes $\mathrm{ACTB}$ and GAPDH to give a relative expression level.

\section{Immunocytochemistry}

Immunocytochemistry was performed as previously described [29] on cells on Geltrex-coated glass coverslips. Cells were washed once with warmed basal medium (DMEM/F12 or Neurobasal Medium) and fixed with $4 \%$ paraformaldehyde in DMEM/F12 for $20 \mathrm{~min}$ at room temperature or at $4{ }^{\circ} \mathrm{C}$ overnight. Fixed cells were then washed three times with PBS and blocked using a buffer containing 10\% normal goat serum (NGS) for $1 \mathrm{~h}$ at room temperature. Primary antibody was diluted in incubation buffer containing $0.1 \%$ Triton $\mathrm{X}-100$ in PBS and $1 \% \mathrm{NGS}$, and incubation was performed at $4{ }^{\circ} \mathrm{C}$ overnight or $2 \mathrm{~h}$ at room temperature. Cells were then washed again with PBS three times, and secondary antibody was diluted in incubation buffer. Secondary antibody incubation was performed for $1 \mathrm{~h}$ at room temperature. Cells were then washed twice with PBS and mounted onto slides with Mowiol and allowed to dry at room temperature overnight before imaging. Alexa fluorconjugated secondary antibodies (Life Technologies) were used for single and double labeling, and all secondary antibodies were tested for specificity.

The primary antibodies used are rat monoclonal antiCD44 at 1:1000 dilution (ThermoFisher Scientific, Waltham, MA); rabbit polyclonal anti-GFAP antibody at 1 : 1000 dilution (DAKO, Glostrup, Denmark); mouse monoclonal anti-S100 $\beta$ at 1:1000 dilution (MilliporeSigma); rabbit anti-VIMENTIN at 1: 250 dilution (Cell Sig. Tech., Danvers, MA); and rabbit anti-NF-k $\beta$ at 1 : 1000 dilution (Cell Sig. Tech.). Secondary antibodies used are Alexa Fluor 555 Goat Anti-Mouse and Alexa Fluor 488 Goat Anti-Rabbit (Life Technologies; Carlsbad, CA). DAPI (Molecular Probes, Thermo Fisher) at 1 : 2000 dilution was used for nuclei staining. Images were captured using Zeiss 700 and 710 Laser Scanning Confocal Microscopes (Zeiss, Thornwood, NY).

\section{Glutamate uptake assay}

$1 \times 10^{5}$ NCRM-1 astrocytes were plated per well of a 24well plate and treated with TNF $\alpha$ or IL-1 $\beta$ as described. To test for glutamate uptake in astrocyte cultures, the media was removed and astrocytes equilibrated in prewarmed Hanks balanced salt solution (HBSS) for $10 \mathrm{~min}$ at $37^{\circ} \mathrm{C}, 5 \% \mathrm{CO}_{2}$. Astrocytes were then incubated for $1 \mathrm{~h}$ at $37^{\circ} \mathrm{C}, 5 \% \quad \mathrm{CO}_{2}$ in $\mathrm{HBSS} / 0.1 \mathrm{mM}$ L-glutamate (Abcam). Fifty microliters of HBSS/L-glutamate was then removed and the glutamate content determined using a Glutamate Assay Kit (Abcam) according to the manufacturer's instructions. Glutamate uptake data were collected from three biological replicates.

\section{Phagocytosis assay}

Red (Ex580/Em605) carboxylate-modified microspheres (FluoSpheres, F8821, ThermoFisher Scientific) were obtained and prepared as previously described [30]. Briefly, FluoSpheres were pelleted at $10,000 \mathrm{~g}$ for $15 \mathrm{~min}$ at room temperature. Supernatant was removed, and the pellet was washed in pure water and pelleted again. Following, water was removed and FluoSpheres were re-suspended (3\% BSA, $25 \mathrm{mM} \mathrm{Na}_{2} \mathrm{HPO}_{4}, \mathrm{pH} 6.0$ ) by rapid vortexing.

$5 \times 10^{5}$ NCRM-1 astrocytes were plated per well of a six-well plate and cultures treated with $100 \mu \mathrm{g} / \mathrm{ml} \mathrm{TNF} \alpha$, IL-1 $\beta$, or with PBS/BSA vehicle control for 7 days (the media/cytokine/vehicle was refreshed after 4 days). Fifteen microliters of beads were added directly to each well for $30 \mathrm{~min}$ at $37^{\circ} \mathrm{C}$. To collect cells for analysis, media was removed and cells were washed twice with PBS, then gently scraped and centrifuged at $300 \mathrm{~g}$ for 10 min. Cell pellets were re-suspended in FACS buffer and submitted to flow cytometry (BD Accuri C6, BD Biosciences, Franklin Lakes, NJ). Cells were gated so that only viable cells were counted. Ten thousand cells per treatment group were counted. To adjust for background, control cells that did not have beads were used for each experiment. The mean fluorescence of all viable cells was measured, and data were analyzed using unpaired Student's $t$ tests. 


\section{Data analysis and statistics}

All data are presented as mean \pm standard deviation of the mean unless otherwise stated. All data was using one-way ANOVA with Sidak's or Tukey's multiple comparisons test or by unpaired Student's $t$ test (GraphPad Prism). A $p$ value of $<0.05$ was considered significant.

\section{Results}

\section{Human NCRM-1 NSPCs can be rapidly differentiated to} TNFa sensitive astrocytes

In order to test the inflammatory responses of human astrocytes, we differentiated astrocytes from NCRM-1 NSPCs as described previously [16, 17]. This protocol results in the rapid differentiation of NSPCs to CD44+/ GFAP/S100 $\beta+/$ VIMENTIN + astrocytes within 35-50 days (Fig. 1a). RNA-seq profiling of astrocytes reveals the upregulation of astrocytic and downregulation of neuronal genes compared to NSPCs and an immature astrocytic gene signature more consistent with human fetal astrocyte progenitors (APCs) than mature astrocytes, see [17].

We next treated NCRM-1 astrocytes with pro-inflammatory cytokines TNF $\alpha$ or IL- $1 \beta$ for 7 days in culture to examine the effects of chronic treatment on cell signaling. Cytokines were added to cell culture media at concentrations of 10,30 , or $100 \mathrm{ng} / \mathrm{mL}$. Western blots demonstrate a significant $(p<0.05)$ increase in phosphorylation of the transcription factor NF- $k \beta$ with 30 and $100 \mathrm{ng} / \mathrm{mL}$ TNF $\alpha$ treatment (Fig. 1b). Increased NF$\kappa \beta$ phosphorylation is detected at $24 \mathrm{~h}$ with $100 \mathrm{ng} / \mathrm{mL}$ TNF $\alpha$ treatment, and immunocytochemistry reveals increased NF- $\kappa \beta$ nuclear localization with TNF $\alpha$ treatment compared to vehicle controls (Fig. 1c). In contrast, IL$1 \beta$-treated phospho-NF- $\kappa \beta$ levels were statistically unchanged when compared to vehicle controls (Fig. 1b) and NF-kB nuclear localization noticeably lower when compared to TNFo-treated cultures (Fig. 1c). Elevated phosphorylation and nuclear localization of NF- $\kappa \beta$ are consistent with activated NF- $\mathrm{k} \beta$ signaling in NCRM-1 astrocytes in response to chronic TNF $\alpha$ treatment. In contrast, the data indicate NF- $\mathrm{k} \beta$ signaling is unaffected or attenuated (to below detection levels) with IL-1 $\beta$ treatment of NCRM-1 astrocytes.

\section{TNFa suppresses glutamate uptake and enhances} phagocytic activity in human NCRM-1 astrocytes

We next examined the effects of chronic TNF $\alpha$ or IL-1 $\beta$ treatment on homeostatic functions of NCRM-1 astrocytes. Astrocytes play a central role in establishing extracellular levels of the excitatory neurotransmitter glutamate in the CNS via active glutamate uptake by excitatory amino acid transporter 1 and 2 (EAAT1 and EAAT2) [31]. We tested the effects of chronic cytokine treatment on glutamate uptake by NCRM-1 astrocytes.
Following 7-day cytokine $(10,30$ or $100 \mathrm{ng} / \mathrm{mL})$ or vehicle treatment, astrocytes were incubated for $1 \mathrm{~h}$ in Hanks balanced salt solution (HBSS) of $0.1 \mathrm{mM}$ L-glutamate. HBSS glutamate levels were then measured by colorimetric assay to quantify the amount of glutamate uptake. The assay reveals a significant $(p<0.05$ to $p<$ 0.01 ) dose-dependent reduction in glutamate uptake with TNF $\alpha$ treatment (Fig. 2a). In contrast glutamate uptake was not significantly reduced by IL- $1 \beta$, although mean uptake values were marginally decreased with increasing IL-1 $\beta$ dose indicating a possible attenuated dose-dependent response (Fig. 2a).

Recent evidence has revealed phagocytic activity by astrocytes. Astrocytes actively engulf synapses in the developing and adult brain in neural circuit refinement [32] and engulf cell debris in CNS trauma and disease [33, 34]. We used fluorescent microbeads and flow cytometry to measure phagocytosis by NCRM-1 astrocytes following chronic treatment of $100 \mathrm{ng} / \mathrm{mL}$ TNF $\alpha$ or IL-1 $\beta$ compared to vehicle control. The data revealed increased phagocytic activity in TNF $\alpha$ but not IL-1 $\beta$ treated cultures (Fig. $2 b$ ). TNF $\alpha$ significantly increased the percentage of phagocytic cells $(p<0.01)$ and the mean fluorescence intensity (MFI) of cells $(p<0.05)$ indicating an increased number of phagocytic astrocytes and increased phagocytic activity of individual astrocytes in response to TNF $\alpha$. In contrast, IL$1 \beta$ had no significant effect of the percentage or MFI of NCRM-1 astrocytes compared to vehicle controls.

\section{Cytokines TNFa and IL-1 $\beta$ both upregulate pro- inflammatory genes but by widely different magnitudes} Having established TNF $\alpha$ effects on NCRM-1 astrocyte function are consistent with a neuroinflammatory astroglia response, we next performed an unbiased wholetranscriptomics analysis of cytokine-treated astrocytes to further investigate neuroinflammatory gene signatures. NCRM-1 astrocytes were chronically treated with 100 $n g / m L ~ T N F ~ \alpha, I L-1 \beta$, or vehicle controls $(n=3)$. mRNA libraries were generated, clustered, and sequenced, and differential gene expression analysis was performed. Unsupervised hierarchical clustering revealed TNF $\alpha$-treated astrocytes clustered separately, while IL-1 $\beta$-treated samples clustered together with the vehicle-treated NCRM-1 passage control (Fig. 3a). Differential expression analysis of the 19,563 transcripts expressed in NCRM-1 astrocytes (Fragments Per Kilobase of transcript per Million mapped reads, FPKM >1) resulted in the identification of 461 genes significantly deregulated with TNF $\alpha$ treatment compared to vehicle controls $(p<0.05$ and $\log$ FC $>1$ ). In contrast, IL-1 $\beta$ treatment resulted in only 33 significantly differentially expressed genes compared to vehicle controls, all of which are activated. Notably, a cross-comparison of genes significantly upregulated by IL-1 $\beta$ and TNF $\alpha$ treatments reveals a large overlap 

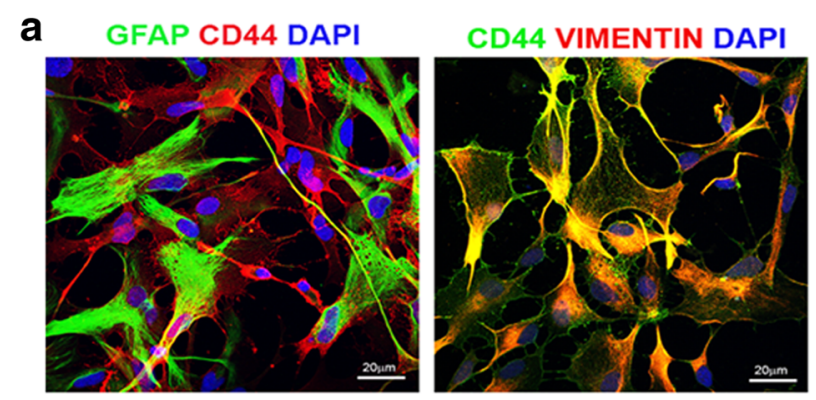

\section{GFAP $S 100 \beta$ DAPI}
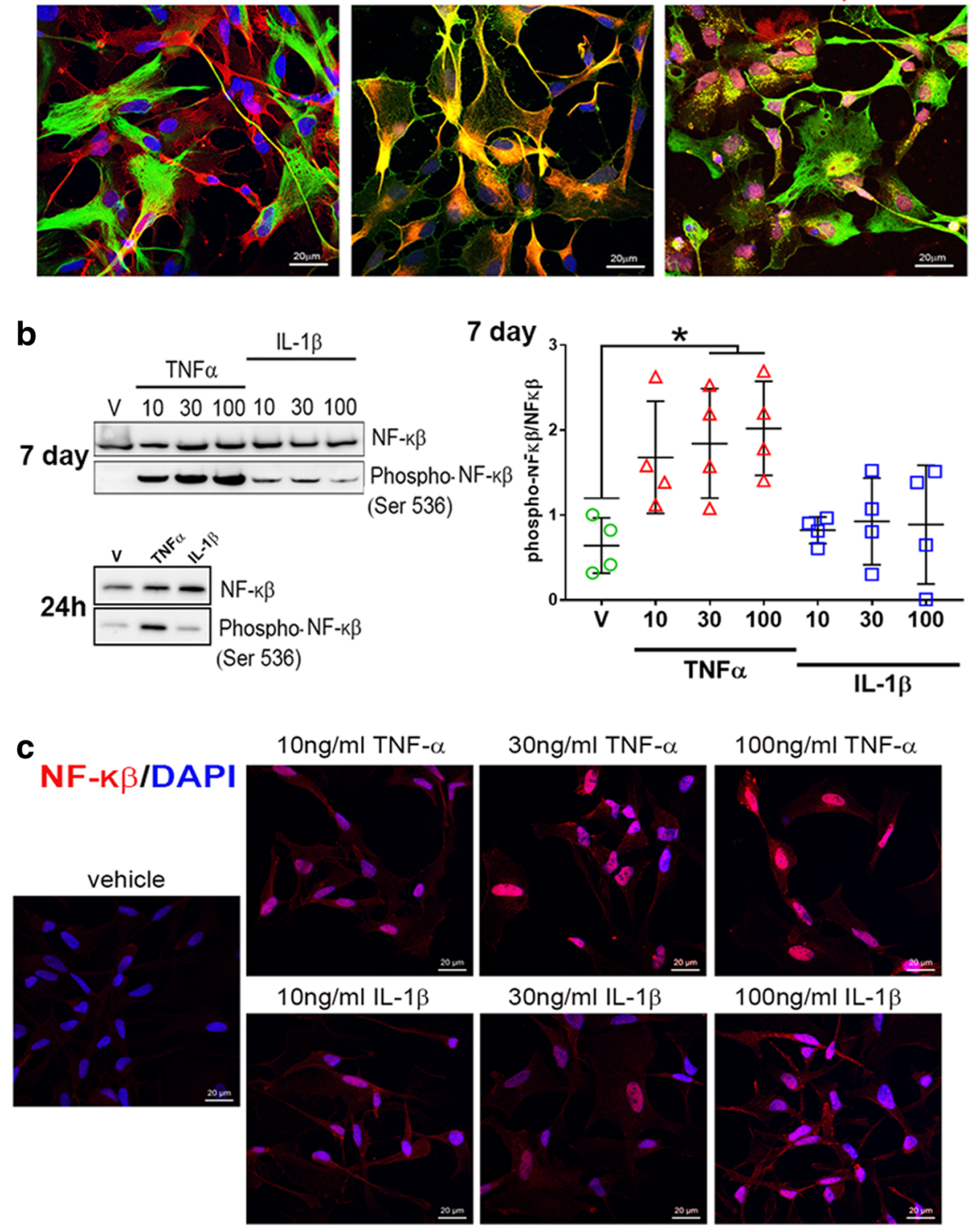

Fig. 1 Differentiated astrocytes from human stem cells respond to cytokines. a NCRM-1 iPSC-derived NSPCs differentiated to astrocytes for 5-7 weeks in vitro express glial cell lineage markers CD44, GFAP, VIMENTIN, and S100ß. b Chronic TNFa but not IL-1 $\beta$ treatment results in a significant dose-dependent increase in NF-K $\beta$ phosphorylation measured by Western blot densitometry. ${ }^{*} p<0.05$ one-way ANOVA with Tukey's multiple comparisons test. Data are presented as mean $\pm \mathrm{SD}, n=3$. c Anti-NF-K $\beta$ immunocytochemistry reveals increased NF-k $\beta$ nuclear localization with chronic TNFa treatment

between IL-1 $\beta$-activated and TNF $\alpha$-activated gene datasets: almost all IL-1 $\beta$-upregulated genes (28 out of 33 ) were also upregulated by TNF $\alpha$ (Fig. 3b). Genes highly upregulated by both TNF $\alpha$ and IL-1 $\beta$ treatment include the complement gene $\mathrm{C} 3$, chemokine genes CXCL10 and CXCL11, and the cytokine gene IL8 (Fig. 3c), consistent with both cytokines eliciting a neuroinflammatory response.

The greater number of differentially expressed genes with TNF $\alpha$ compared to IL- $1 \beta$ is consistent with a more widespread transcriptional response to this cytokine in differentiated astrocytes. Our whole-transcriptomics analysis therefore supports a more robust neuroinflammatory transcriptional response to TNF $\alpha$ compared to IL-1 $\beta$. These results were independently validated by an orthogonal assay using qRT-PCR of a selected neuroinflammatory gene cohort. We confirmed that six neuroinflammatory genes (C3, CCL2, CXCL10, CXCL11, IL8, and MMP9) showed a greater gene upregulation by orders of magnitude in response to TNF $\alpha$ compared to IL$1 \beta$ treatment (Fig. 3d, e). We next examined upregulated 

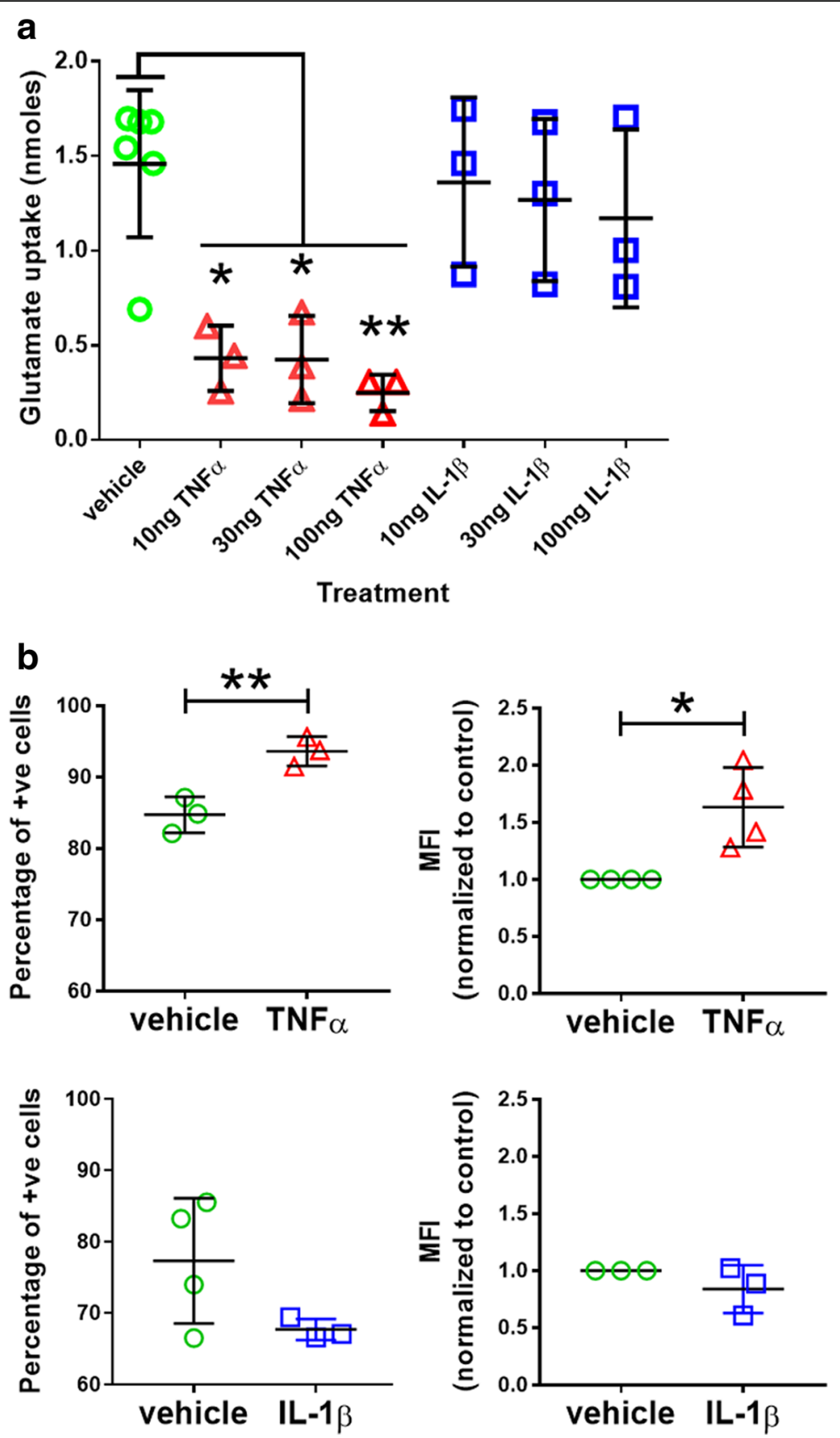

Fig. 2 TNFa but not IL-1 $\beta$ treatment suppresses glutamate uptake and enhances phagocytic activity by differentiated astrocytes. a Glutamate uptake by astrocyte cultures is significantly reduced following chronic treatment with TNFa but not IL-1 $\beta\left({ }^{* *} p<0.01,{ }^{*} p<0.05\right.$ one-way ANOVA with Tukey's multiple comparisons test, cytokine $n=3$, vehicle $n=6$ ). $\mathbf{b}$ The number of phagocytic astrocytes (measured as the percent of fluorescent positive cells) and the rate of phagocytosis (measured as the mean fluorescent intensity, MFI) are significantly increased by TNFa but not IL-1 $\beta .{ }^{* *} p<0.01,{ }^{*} p<0.05$ unpaired Student's $t$ test, $n=3-4$. Data are presented as mean \pm SD

gene pathways by unbiased gene ontology (GO). GO searches reveal gene cohorts functioning in host defense to pathogens or injury, cytokine production, extracellular function, and the regulation of I- $\kappa \beta$ kinase/NF- $k \beta$ cascade are activated by TNF $\alpha$ (Table 2 ). IL- $1 \beta$ similarly activates $\mathrm{GO}$ gene cohorts functioning in host defense to pathogens or injury as well as genes functioning in antigen presentation and leukocyte migration (Table 2). Taken together, the RNA sequencing data reveals a neuroinflammatory transcriptional response to TNF $\alpha$ and IL-1 $\beta$ in differentiated astrocytes. The number of differential expressed genes and the magnitude of 
a
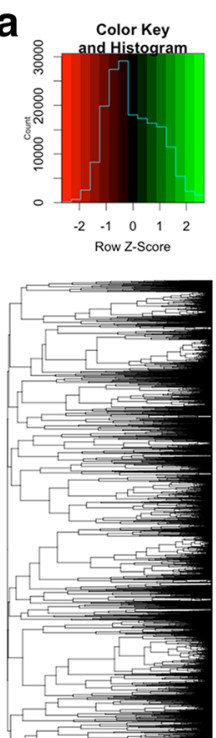

$\square$
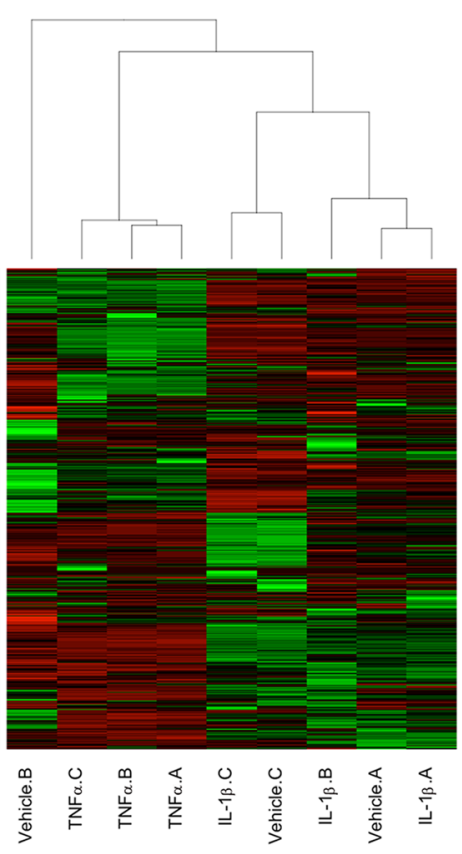

b

\section{$\geq 2$-fold upregulated genes}
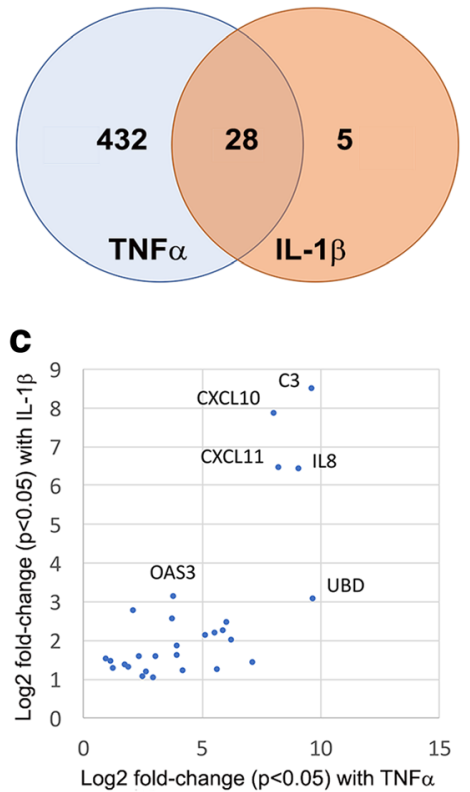
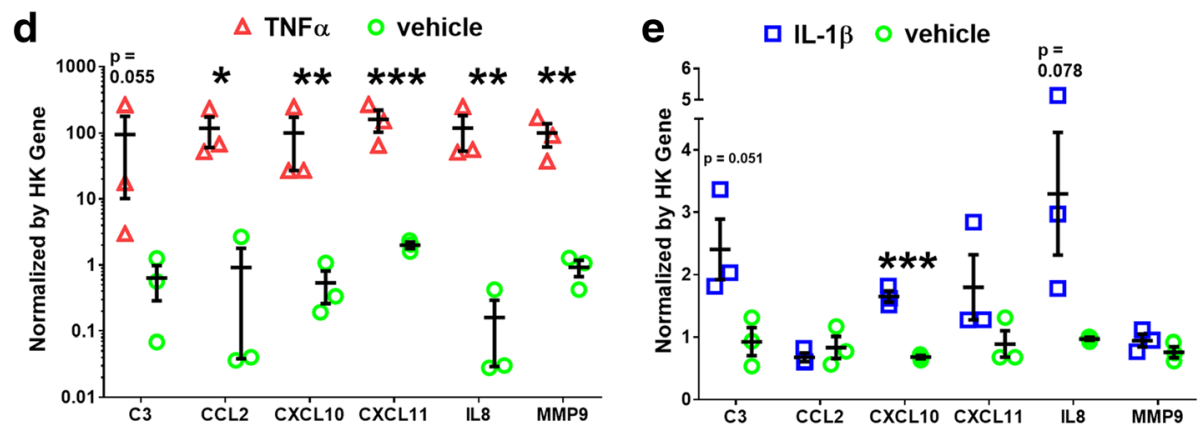

Fig. 3 RNA sequencing reveals TNFa and IL-1 $\beta$ treatment result in the activation of neuroinflammatory genes with different effect sizes. a Unsupervised hierarchical clustering of the whole transcriptome of TNFa-, IL-1 3 -, and vehicle-treated differentiated astrocytes clusters TNFa samples by treatment. In contrast, the majority of IL-1 $\beta$ samples cluster more closely to the same passage vehicle control. b Venn diagram of $\geq+2$-fold change reveals differential gene activation effect sizes but overlapping upregulated genes by TNFa and IL-1 $\beta$ treatment. c Genes highly upregulated by either TNFa or IL-1 $\beta$ treatment have neuroinflammatory functions. $\mathbf{d}$ Independent verification of a cohort of six TNFa activated genes by qRT-PCR expression screening following 7 days TNFa treatment. e Independent qRT-PCR expression screening of the same six gene cohort following 7 days IL-1 $\beta$ treatment. Although IL-1 $\beta$ results in elevated mRNA levels for all six genes relative to control, the magnitude of increased expression is considerably reduced when compared to the TNFa qRT-PCR dataset (note $\log 10$ scale for TNFa compared to linear scale for $\mathrm{IL}-1 \beta$ data). ${ }^{* * *} p<0.001,{ }^{* *} p<0.01,{ }^{*} p<0.05$ unpaired Students $t$ test, one-way ANOVA with Sidak's multiple comparisons test $n=3$. Data are presented as mean \pm SD

neuroinflammatory gene activation changes are greater in response to TNF $\alpha$ compared to IL-1 $\beta$ treatment consistent with the greater effect of TNF $\alpha$ on $N F-\kappa \beta$ signaling, glutamate uptake, and phagocytosis.

Next, we examined the RNA-seq data for a possible explanation for the differential effects of TNF $\alpha$ compared to IL-1 $\beta$ on differentiated astrocytes. Messenger RNA transcript levels (Fragments Per Kilobase of transcript per Million mapped reads) are consistent with greater expression of TNF $\alpha$ compared to IL- $1 \beta$ receptor-signaling genes in NCRM-1 astrocytes (not shown). Higher mRNA levels of the canonical TNF $\alpha$ receptor gene TNFR1 (gene name TNFRSF1A) compared to the canonical IL-1 $\beta$ receptor IL1R1 were confirmed by qRT-PCR (Fig. 4a), and Western blots using antibodies to the same canonical TNF $\alpha$ and IL$1 \beta$ receptors reveal comparatively greater expression of TNFR1 compared to IL1R1 protein in NCRM-1 astrocytes (Fig. 4b). Thus, differences in the expression levels of canonical receptors for TNF $\alpha$ and IL-1 $\beta$ could account for the differential effects of these cytokines on differentiated NCRM-1 astrocytes. 
Table 2 Gene ontology (GO) analysis for all significantly upregulated genes by chronic treatment with TNFa (2122 genes) or II-1 $\beta$ (156 genes) using DAVID. Listed is the highest representative GO annotation term by false discovery rate (FDR) for each of the four highest clusters ranked by cluster enrichment score. Also listed is the total number of genes for each annotation category, the percentage of TNFa or II-1 $\beta$ upregulated genes within this annotation, the fold-enrichment of this GO gene set, and the FDR corrected $p$ values for each GO enrichment

\begin{tabular}{|c|c|c|c|c|c|c|}
\hline \multicolumn{7}{|c|}{ Genes upregulated with TNFa treatment } \\
\hline Cluster & $\begin{array}{l}\text { Cluster enrichment } \\
\text { score }\end{array}$ & GO term & $\begin{array}{l}\text { Total number of } \\
\text { genes in category }\end{array}$ & $\begin{array}{l}\% \text { of TNFa upregulated } \\
\text { genes in category }\end{array}$ & $\begin{array}{l}\text { Fold } \\
\text { enrichment }\end{array}$ & FDR \\
\hline 1 & 8.81 & GO:0006952 defense response & 118 & 5.98 & 1.75 & $\begin{array}{l}1.77 \mathrm{E} \\
-06\end{array}$ \\
\hline 2 & 6.61 & $\begin{array}{l}\text { GO:0001817 regulation of cytokine } \\
\text { production }\end{array}$ & 50 & 2.54 & 2.52 & $\begin{array}{l}2.02 E \\
-06\end{array}$ \\
\hline 3 & 6.58 & GO:0044421 extracellular region part & 159 & 8.06 & 1.59 & $\begin{array}{l}2.14 \mathrm{E} \\
-06\end{array}$ \\
\hline 4 & 5.57 & $\begin{array}{l}\text { GO:0043122 regulation of I-kappaB kinase/ } \\
\text { NF-kappaB cascade }\end{array}$ & 32 & 1.62 & 2.73 & $\begin{array}{l}4.43 \mathrm{E} \\
-04\end{array}$ \\
\hline \multicolumn{7}{|c|}{ Genes Upregulated with IL-1 $\beta$ treatment } \\
\hline Cluster & $\begin{array}{l}\text { Cluster enrichment } \\
\text { score }\end{array}$ & GO Term & $\begin{array}{l}\text { Total number of } \\
\text { genes in category }\end{array}$ & $\begin{array}{l}\% \text { of } I L-1 \beta \text { upregulated } \\
\text { genes in category }\end{array}$ & $\begin{array}{l}\text { Fold } \\
\text { enrichment }\end{array}$ & FDR \\
\hline 1 & 4.77 & GO:0006952 defense response & 25 & 17.36 & 4.70 & $\begin{array}{l}5.451 \mathrm{E} \\
-07\end{array}$ \\
\hline 2 & 3.30 & $\begin{array}{l}\text { GO:0019882 antigen processing and } \\
\text { presentation }\end{array}$ & 11 & 7.64 & 15.32 & $\begin{array}{l}3.451 \mathrm{E} \\
-06\end{array}$ \\
\hline 3 & 2.39 & GO:0050900 leukocyte migration & 7 & 4.86 & 4.20 & 0.01 \\
\hline 4 & 2.19 & $\begin{array}{l}\text { GO:0070011 peptidase activity, acting on L- } \\
\text { amino acid peptides }\end{array}$ & 12 & 8.33 & 2.68 & 6.12 \\
\hline
\end{tabular}

\section{Discussion}

The development of human stem cell culture models of neuroinflammatory astrocytes is expected to advance our understanding of the pathological roles of this cell type in CNS trauma, infection, and disease. In this study, we demonstrate human iPSC-derived NSPCs can be rapidly differentiated to neuroinflammatory reactive astrocytes. We show chronic TNF $\alpha$ treatment in culture activated NF- $\mathrm{N} \beta$ signaling in differentiated astrocytes to depress glutamate uptake, increase phagocytic activity, and trigger widespread changes in gene expression that included the activation of classical pro-inflammatory gene targets. These data demonstrate our iPSC-derived astrocyte model provides an easily accessible human cell culture system for investigating TNF $\alpha$ reactive astroglia in CNS injury, infection, and disease.

Reactive astrogliosis in the CNS has recently been revealed to be a divergent stimulus-dependent cell state. Reactive astrocytes in lipopolysaccharide (LPS) injection models of systemic infection are neurotoxic (referred to as $\mathrm{A} 1$ astrocytes), whereas reactive astrocytes in a stroke model of middle cerebral artery occlusion (MCAO) are
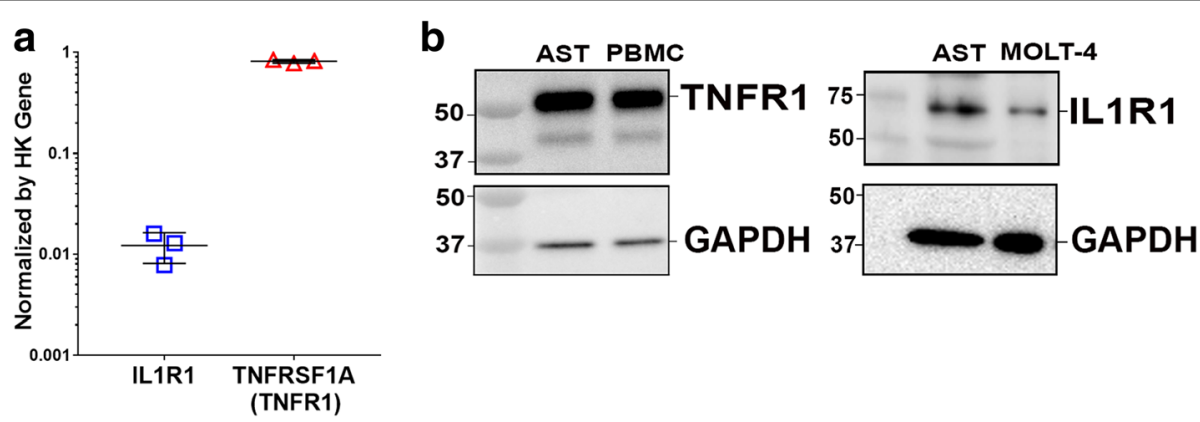

Fig. 4 Expression analysis reveals comparatively higher TNFa compared to IL-1 $\beta$ receptor expression in differentiated astrocytes. a qRT-PCR confirms higher mRNA levels for canonical TNFa receptor gene TNFR1 (TNFRSF1A) compared to canonical IL-1 $\beta$ receptor IL1R1 in differentiated astrocytes. b Western blot confirms higher TNFR1 compared to IL1R1 protein expression in differentiated astrocytes (AST). Peripheral blood mononuclear cells (PBMC), and MOLT-4 cell lysates were included as positive TNFR1 and IL1R1 controls respectively 
neuroprotective (referred to as A2 astrocytes) [13]. A1 reactive astrocytes are induced by reactive microglia, and screening identifies the candidate microglia proteins TNF $\alpha$, IL- $1 \alpha$, and C1q as the strongest inducers of a partial A1 astrocyte phenotype [13]. LPS significantly increases the secretion of TNF $\alpha$, IL- $1 \alpha$, and C1q by microglia in vitro, confirming these three microglia-derived proteins are key drivers of A1 astrocytic states. LPS-activated microglia also secrete IL-1 $\beta$, but the cytokine is not able to induce A1 transcripts indicating a prominent TNF $\alpha$ but not IL-1 $\beta$ signaling component in A1 astrogliosis. Accordingly, we speculate chronic TNF $\alpha$ treatment induces a partial A1 astroglial fate in NCRM-1 astrocytes. Interestingly, it should be noted we observed increased general phagocytic activity with TNF $\alpha$ treatment in contrast to decreased synaptosome phagocytosis reported in A1 astrocytes [13]. It is feasible that the physiological removal of synaptosomes compared to the pathological removal of cell debris (modeled in our assay) are differentially affected by A1 induction consistent with this disparity in astrocyte phagocytosis data. However, this remains to be demonstrated.

In contrast to the robust response to $\mathrm{TNF} \alpha$, astrocyte responses to IL-1 $\beta$ were attenuated. Although chronic IL-1 $\beta$ treatment similarly elicited pro-inflammatory gene upregulation, the scope and magnitude of gene changes were substantially reduced when compared to TNF $\alpha$ treated astrocytes. Furthermore, IL-1 $\beta$ did not significantly activate NF- $\mathrm{k} \beta$ signaling or alter glutamate uptake or phagocytosis in our in vitro assays. We provide transcriptome data and Western blot evidence this attenuated response could be due to comparatively lower levels of the cognate receptor for IL-1 $\beta$ in our differentiated astrocytes.

NSPCs cultured under our astrocyte culture conditions rapidly develop CD44 antigen immunoreactivity within the first 1-3 weeks, assume a fibrous or protoplasmic-like morphology, and express multiple glial cell marker proteins after $\approx 35$ days differentiation [17]. Despite the expression of astrocyte markers such as GFAP, S100 $\beta$, and VIMENTIN, RNA-seq expression screening data of differentiated NCRM-1 astrocytes is more consistent with an immature astrocyte progenitor cell (APC) state than a mature astrocyte cell status [17]. It is therefore possible that an immature APC-like status of our differentiated astrocytes could account for the attenuated response to IL-1 $\beta$. Another possibility is that cell culture artifacts are responsible. A recent 2017 study of inflammation-responsive human iPSC-derived astrocytes reported strong upregulation of pro-inflammatory genes in response to IL-1 $\beta$ [17]. In this study, astrocytes were differentiated using a neuronal medium with leukemia inhibitory factor (LIF) and serum. Our astrocytes were differentiated from iPSC-derived NSPCs in media containing the growth factor Heregulin and the TGF-beta superfamily member Activin A with serum [16]. Although there is signaling convergence between these two astrocyte protocols with both Heregulin and LIF signaling through JAK/STAT3 and PI3/AKT pathways and Activin enhancement of LIF-mediated astrocyte differentiation [35], it is highly likely the two protocols generate transcriptionally distinct astrocytes. Furthermore, the NSPCs differentiated to astrocytes in our study are positionally naïve, whereas the 2017 study used dorsal forebrain NSPCs following SMAD inhibition patterning. Thus, our cultures represent a highly rapid system for modeling A1-like TNFo-responsive astroglial states but may not be applicable to astrogliosis in all neuroinflammatory conditions or CNS regions.

In this study, we highlight the benefits of a readily accessible cell source, relatively simple technical procedures, and high reproducibility of iPSC-based approaches to model human astrocyte pathology in the laboratory. However, it should be noted that the differentiation of astrocytes from progenitors in cell culture has been linked to a reactive astroglia transcriptome in cultured astrocytes that may blunt or bias experimental outcomes [12]. In contrast, the transcriptome of astrocytes isolated by immuno-panning from surgically resected human brain or fetal tissue is similar to that of in vivo astrocytes and is considered a more physiological astrocyte state [1]. Although this suggests caution should be applied when evaluating iPSC-derived astrocyte studies, stem cell culture methods remain the more practical option for modeling neurological conditions in culture due to the problems of tissue availability for immunopanning combined with the difficulty of replicating disease-related genomic conditions in immuno-isolated astrocytes.

\section{Conclusion}

In conclusion, this manuscript describes a rapid protocol for in vitro modeling neuroinflammatory human astrocytes in CNS trauma or neurological diseases. Using this protocol, NCRM-1 astrocyte can be used for highthrough screens to identify novel anti-inflammatory drugs to treat CNS trauma or disease.

\section{Abbreviations \\ ADM: Astrocyte differentiation medium; CNS: Central nervous system; EB: Embryoid body; FC: Fold change; FPKM: Fragments Per Kilobase of transcript per Million mapped read; GO: Gene ontology; IL-1ß: Interleukin-1 beta; iPSC: Induced pluripotent stem cell; LPS: Lipopolysaccharide; MCA: Middle cerebral artery; MFI: Mean fluorescence intensity; NSPC: Neural stem progenitor cell; TNFa: Tumor necrosis factor alpha}

\section{Acknowledgements}

We thank Andrew Snow for the donation of peripheral blood mononuclear cells (PBMCS).

\section{Authors' contributions}

$\mathrm{QZ}, \mathrm{AE}, \mathrm{GK}$, and KEM prepared the cell cultures and generated in vitro cell assay data. GS prepared RNA sequencing libraries. CV, MW, and CLD 
performed RNA sequencing data output and bioinformatics analysis. MLD conceived, directed, and interpreted the study. MLD authored the manuscript with editorial assistance from CV. All authors read and approved the final manuscript.

\section{Funding}

This work was supported by a grant from the Center for Neuroscience and Regenerative Medicine (CNRM, grant number G170244014).

\section{Availability of data and materials}

The datasets used and/or analyzed during the current study are available from the corresponding author on reasonable request and/or data will be deposited with the Genome Expression Omnibus https://www.ncbi.nlm.nih. gov/geo/

\section{Ethics approval and consent to participate}

Not applicable.

\section{Consent for publication}

Not applicable.

\section{Competing interests}

The authors declare that they have no competing interests.

\section{Author details}

${ }^{1}$ Center for Neuroscience and Regenerative Medicine, Uniformed Services University of the Health Sciences, Bethesda, MD 20814, USA. ${ }^{2}$ Boehringer Ingelheim Pharma GmbH \& Co. KG, Computational Biology, Biberach, Germany. ${ }^{3}$ Ronald M. Loeb Center for Alzheimer's Disease, Icahn School of Medicine at Mount Sinai, New York, NY 10029, USA. ${ }^{4}$ Department of Anatomy, Physiology and Genetics, Center for Neuroscience and Regenerative Medicine, Uniformed Services University of the Health Sciences, Bethesda, MD 20814, USA. ${ }^{5}$ Viral Immunology and Intravital Imaging Section, National Institute of Neurological Disorders and Stroke, National Institutes of Health, Bethesda, MD 20892, USA. ${ }^{6}$ Collaborative Health Initiative Research Program, Uniformed Services University of the Health Sciences, Bethesda, MD 20814, USA.

\section{Received: 13 March 2019 Accepted: 25 July 2019}

Published online: 09 August 2019

\section{References}

1. Zhang Y, Sloan SA, Clarke LE, Caneda C, Plaza CA, Blumenthal PD, et al. Purification and characterization of progenitor and mature human astrocytes reveals transcriptional and functional differences with mouse. Neuron. 2016;89(1):37-53.

2. Allen NJ, Eroglu C. Cell biology of astrocyte-synapse interactions. Neuron. 2017;96(3):697-708.

3. Nortley R, Attwell D. Control of brain energy supply by astrocytes. Curr Opin Neurobiol. 2017;47:80-5

4. Rothstein JD, Dykes-Hoberg M, Pardo CA, Bristol LA, Jin L, Kuncl RW, et al. Knockout of glutamate transporters reveals a major role for astroglial transport in excitotoxicity and clearance of glutamate. Neuron. 1996:16(3):675-86.

5. Attwell D, Buchan AM, Charpak S, Lauritzen M, Macvicar BA, Newman EA. Glial and neuronal control of brain blood flow. Nature. 2010; 468(7321):232-43

6. Cheslow L, Alvarez JI. Glial-endothelial crosstalk regulates blood-brain barrier function. Curr Opin Pharmacol. 2016;26:39-46.

7. Blanco-Suarez E, Caldwell AL, Allen NJ. Role of astrocyte-synapse interactions in CNS disorders. J Physiol. 2017;595(6):1903-16.

8. Chen $C$, Jiang P, Xue H, Peterson SE, Tran HT, McCann AE, et al. Role of astroglia in Down's syndrome revealed by patient-derived humaninduced pluripotent stem cells. Nat Commun. 2014;5:4430.

9. Kisler K, Nelson AR, Montagne A, Zlokovic BV. Cerebral blood flow regulation and neurovascular dysfunction in Alzheimer disease. Nat Rev Neurosci. 2017:18(7):419-34.

10. Yun SP, Kam TI, Panicker N, Kim S, Oh Y, Park JS, et al. Block of A1 astrocyte conversion by microglia is neuroprotective in models of Parkinson's disease. Nat Med. 2018;24(7):931-8.
11. Sofroniew MV. Astrogliosis. Cold Spring Harb Perspect Biol. 2015;7(2): a020420.

12. Zamanian JL, Xu L, Foo LC, Nouri N, Zhou L, Giffard RG, et al. Genomic analysis of reactive astrogliosis. J Neurosci. 2012;32(18):6391-410.

13. Liddelow SA, Guttenplan KA, Clarke LE, Bennett FC, Bohlen CJ, Schirmer $L$, et al. Neurotoxic reactive astrocytes are induced by activated microglia. Nature. 2017;541(7638):481-7.

14. Oberheim NA, Takano T, Han X, He W, Lin JH, Wang F, et al. Uniquely hominid features of adult human astrocytes. J Neurosci. 2009;29(10):3276-87.

15. Han X, Chen M, Wang F, Windrem M, Wang S, Shanz S, et al. Forebrain engraftment by human glial progenitor cells enhances synaptic plasticity and learning in adult mice. Cell Stem Cell. 2013; 12(3):342-53.

16. Shaltouki A, Peng J, Liu Q, Rao MS, Zeng X. Efficient generation of astrocytes from human pluripotent stem cells in defined conditions. Stem Cells. 2013;31(5):941-52.

17. Lischka FW, Efthymiou A, Zhou Q, Nieves MD, McCormack NM, Wilkerson MD, et al. Neonatal mouse cortical but not isogenic human astrocyte feeder layers enhance the functional maturation of induced pluripotent stem cell-derived neurons in culture. Glia. 2018;66(4):725-48.

18. Zhao J, Davis MD, Martens YA, Shinohara M, Graff-Radford NR, Younkin SG, et al. APOE epsilon4/epsilon4 diminishes neurotrophic function of human iPSC-derived astrocytes. Hum Mol Genet. 2017; 26(14):2690-700

19. Hall CE, Yao Z, Choi M, Tyzack GE, Serio A, Luisier $R$, et al. Progressive motor neuron pathology and the role of astrocytes in a human stem cell model of VCP-related ALS. Cell Rep. 2017;19(9):1739-49.

20. Juopperi TA, Kim WR, Chiang $\mathrm{CH}, \mathrm{Yu} \mathrm{H}$, Margolis RL, Ross CA, et al. Astrocytes generated from patient induced pluripotent stem cells recapitulate features of Huntington's disease patient cells. Mol Brain. 2012;5:17.

21. Serio A, Bilican B, Barmada SJ, Ando DM, Zhao C, Siller R, et al. Astrocyte pathology and the absence of non-cell autonomy in an induced pluripotent stem cell model of TDP-43 proteinopathy. Proc Natl Acad Sci U S A. 2013;110(12):4697-702.

22. Chou BK, Mali P, Huang X, Ye Z, Dowey SN, Resar LM, et al. Efficient human iPS cell derivation by a non-integrating plasmid from blood cells with unique epigenetic and gene expression signatures. Cell Res. 2011:21(3):518-29.

23. Swistowski A, Peng J, Han Y, Swistowska AM, Rao MS, Zeng X. Xeno-free defined conditions for culture of human embryonic stem cells, neural stem cells and dopaminergic neurons derived from them. PLoS One. 2009;4(7):e6233.

24. Efthymiou A, Shaltouki A, Steiner JP, Jha B, Heman-Ackah SM, Swistowski A, et al. Functional screening assays with neurons generated from pluripotent stem cell-derived neural stem cells. J Biomol Screen. 2014;19(1):32-43.

25. Yan $Y$, Shin S, Jha BS, Liu Q, Sheng J, Li F, et al. Efficient and rapid derivation of primitive neural stem cells and generation of brain subtype neurons from human pluripotent stem cells. Stem Cells Transl Med. 2013;2(11):862-70.

26. Reich M, Liefeld T, Gould J, Lerner J, Tamayo P, Mesirov JP. GenePattern 2.0. Nat Genet. 2006;38(5):500-1.

27. Mi H, Muruganujan A, Casagrande JT, Thomas PD. Large-scale gene function analysis with the PANTHER classification system. Nat Protoc. 2013;8(8):1551-66.

28. Huang d W, Sherman BT, Lempicki RA. Systematic and integrative analysis of large gene lists using DAVID bioinformatics resources. Nat Protoc. 2009;4(1):44-57.

29. Efthymiou AG, Steiner J, Pavan WJ, Wincovitch S, Larson DM, Porter $F D$, et al. Rescue of an in vitro neuron phenotype identified in Niemann-Pick disease, type C1 induced pluripotent stem cell-derived neurons by modulating the WNT pathway and calcium signaling. Stem Cells Transl Med. 2015:4(3):230-8.

30. Kopec KK, Carroll RT. Alzheimer's beta-amyloid peptide 1-42 induces a phagocytic response in murine microglia. J Neurochem. 1998;71(5): 2123-31.

31. Verkhratsky A, Nedergaard M. The homeostatic astroglia emerges from evolutionary specialization of neural cells. Philos Trans R Soc Lond Ser B Biol Sci. 2016;371(1700).

32. Chung WS, Clarke LE, Wang GX, Stafford BK, Sher A, Chakraborty C, et al. Astrocytes mediate synapse elimination through MEGF10 and MERTK pathways. Nature. 2013:504(7480):394-400. 
33. Koizumi S, Hirayama Y, Morizawa YM. New roles of reactive astrocytes in the brain; an organizer of cerebral ischemia. Neurochem Int. 2018;119:107-14.

34. Chung WS, Welsh CA, Barres BA, Stevens B. Do glia drive synaptic and cognitive impairment in disease? Nat Neurosci. 2015;18(11):1539-45.

35. Satoh M, Sugino H, Yoshida T. Activin promotes astrocytic differentiation of a multipotent neural stem cell line and an astrocyte progenitor cell line from murine central nervous system. Neurosci Lett. 2000;284(3):143-6.

\section{Publisher's Note}

Springer Nature remains neutral with regard to jurisdictional claims in published maps and institutional affiliations.

Ready to submit your research? Choose BMC and benefit from:

- fast, convenient online submission

- thorough peer review by experienced researchers in your field

- rapid publication on acceptance

- support for research data, including large and complex data types

- gold Open Access which fosters wider collaboration and increased citations

- maximum visibility for your research: over $100 \mathrm{M}$ website views per year

At $\mathrm{BMC}$, research is always in progress.

Learn more biomedcentral.com/submissions 